\title{
Circulating miR-130 is a potential bio signature for early prognosis of acute myocardial infarction
}

\author{
Xiaodong Pan, Yanru He, Zhongpu Chen, Gaoliang Yan, Genshan Ma \\ Department of Cardiology, Zhongda Hospital Affiliated to Southeast University, Nanjing, China \\ Contributions: (I) Conception and design: X Pan; (II) Administrative support: None; (III) Provision of study materials or patients: None; (IV) \\ Collection and assembly of data: All authors; (V) Data analysis and interpretation: All authors; (VI) Manuscript writing: All authors; (VII) Final \\ approval of manuscript: All authors. \\ Correspondence to: Xiaodong Pan. Department of Cardiology, Zhongda Hospital Affiliated to Southeast University, Nanjing, China. \\ Email: panxiaodongseu@163.com.
}

\begin{abstract}
Background: As distinctive leading reasons of death globally, acute myocardial infarction (AMI). Accounts for major death ratio, caused by coronary artery disease (CAD). Its diagnosis relies on the presenting clinical symptoms, electrocardiograms (ECGs), and levels of circulating biomarkers. Recent studies have implicated microRNAs (miRNAs) in the pathogenesis of many diseases, including AMI. The present study inquire into feature value of miR-130 in AMI patients.

Methods: levels of expression of miR-130 in patient plasma, considered through simultaneous quantitative polymerase chain reaction (qRT-PCR). The method used for determining Plasma cardiac troponin I (cTnI) \& creatine kinase-MB(CK-MB) degree set on by enzyme-linked immunosorbent assay (ELISA). The diagnostic value of miR-130 was measured using a receiver operating characteristic (ROC) curve.

Results: Plasma miR-130, cTnI, and CK-MB levels exist remarkably inflated in the AMI classification in comparison with control category $(\mathrm{P}<0.05)$. MiR-130 expression peaked 6 hours after disease onset, earlier than cTnI and CK-MB. The level of expression of miR-130 6 hours after disease onset was positively correlated with cTnI and CK-MB levels 12 hours after onset. The optimal cut-off point for miR-130 in peripheral blood, sensitivity, and specificity were $1.58 \mathrm{ng} / \mathrm{mL}, 82.5 \%$ and $77.5 \%$, respectively. The area under curve (AUC) was 0.922 .
\end{abstract}

Conclusions: These results indicate that circulating miR-130 holds great promise as an effective biomarker for diagnosing AMI earlier.

Keywords: Acute myocardial infarction (AMI); cardiac troponin I (cTnI); creatine kinase; diagnosis; MicroRNA-130 (miRNAs)

Submitted Sep 27, 2020. Accepted for publication Dec 09, 2020.

doi: $10.21037 /$ jtd-20-3207

View this article at: http://dx.doi.org/10.21037/jtd-20-3207

\section{Introduction}

Acute myocardial infarction (AMI), a global leading source of mortality, interpretation the mortality caused by coronary artery disease (CAD) (1,2). Early diagnosis of AMI is crucial for effective treatment (3). The diagnosis of AMI relies on the presenting clinical symptoms, electrocardiograms (ECGs), and levels of circulating biomarkers $(4,5)$. As a circulating biomarker for myocardial damage, cardiac- specific troponin (cTn) levels increase rapidly as early as 4 hours after disease onset, but its clinical effectiveness is limited by the delay in the timing of troponin release $(6,7)$. This has necessitated the search for novel biomarkers that are more specific and sensitive, and can overcome this limitation.

MicroRNAs (miRNAs) are a gathering of domestic \& small no encrypting RNAs that commands the appearance of supportive target mRNAs. MicroRNAs (miRNAs) are 
19-22 nucleotides in length, and modulate gene expression on post-arrangements extent by targeting the 3 ' unremitted district (3'UTR) in the organic phenomenon (8). They've been implicated in angiogenesis, CAD, hypertension, atrial fibrillation, cardiomyopathy, dyslipidemia, myocardial infarction, heart failure and stroke $(9,10)$. Demonstration states that extracellular circulation miRNAs are steady in body liquids, making them potential and emerging biomarkers for non-invasive diagnosis. For example, in a previous study, it described that circulating miR-1 levels crucially increases the patients in AMI, and positively correlated with plasma creatine kinase (CK)-MB levels (11). It has been shown that miR-19a has higher diagnostic value than $\mathrm{CK}, \mathrm{CK}-\mathrm{MB}$, myoglobin, high-sensitive troponin (hs-cTn), and brain natriuretic peptide (12). Similarly, miR-449 has been reported to considerably expand the indicating precision of CK-MB and cardiac troponin I (cTnI), therefore acting in a useful marker for the premature identification of AMI (13). The diagnostic and prognostic value of miR-208b in AMI has also been reported (14). The level of expression of miR-208b has been demonstrated to be significantly lower after percutaneous coronary intervention (PCI) than on admission. However, its level is materially elevated in AMI patients along left ventricular remodeling in comparison without left ventricular remodeling after PCI. MiR-130 has been shown to aggravate AMI-induced myocardial injury in H9c2 cells by directly targeting peroxisome proliferator-activated receptor gamma (PPAR- $\gamma$ ) (15). Therefore, this study investigated the diagnostic value of miR-130 in patients with AMI. We present the following article in accordance with the STARD reporting checklist (available at http://dx.doi.org/10.21037/ jtd-20-3207).

\section{Methods}

\section{Materials}

Trizol reagent attained from Invitrogen (USA). Commanding Script II 1st strand cDNA integrate tools and SYBR Premix ExTaq kits, all brought by Takara (China). Enzyme-linked immunosorbent assay (ELISA) kits were products of Roche (Switzerland), while ABI PRISM 7500 sequence detection system was purchased from Applied Biosystems (China).

\section{Patients and general information}

Patients with AMI who received PCI $(\mathrm{n}=80)$ were recruited over a 2 years period for this study. The analytic criteria were created on the universal definition of myocardial infarction mutually recognized by the the American Heart Association, the European Society of Cardiology, World Heart Federation and the American College of Cardiology Foundation (16). Patients with chronic kidney and/or liver diseases are excluded from the study. The control group comprised 40 healthy individuals. All procedures performed in this study involving human participants were in accordance with the Declaration of Helsinki (as revised in 2013). The study protocol was approved by the Clinical Research Ethics Committee of Zhongda Hospital Affiliated to Southeast University, China. The patients signed written informed consent with their family members.

\section{Blood sample collection and preparation of plasma}

Peripheral venous blood $(10 \mathrm{~mL})$ was drawn from the patients in EDTA tubes at different time points: 0 hours on admission and 6,12, 24, and 48 hours after the onset of AMI. At 3,000 rpm the blood was centrifuged 4 degree centigrade for 10 minutes to get plasma. Aliquots of the plasma $(200 \mu \mathrm{L})$ were immediately used for DNA extraction, or kept at -80 degree centigrade before usage.

\section{Quantitative back arrangement polymerase chain reaction (qRT-PCR)}

The level of expression of miR-130 was determined using qRT-PCR. To draw out entire RNA from plasma samples from each group, Trizol RNA extraction reagent suited. According to the manufacturers, instructions performing the combining reaction cDNA, combination kit optimized. Light Cycler 1536 RT-PCR noticing structure was adapted for measuring the expression level of miR-130. Variation in the cDNA content was normalized using glyceraldehyde-3phosphate dehydrogenase (GAPDH).

The qRT-PCR reaction conditions were: pre-denaturation at $95^{\circ} \mathrm{C}$ for $5 \mathrm{~min}, \mathrm{PCR}$ reaction at $95^{\circ} \mathrm{C}$ for $5 \mathrm{sec}$ and $60{ }^{\circ} \mathrm{C}$ for $30 \mathrm{sec}$, and a total of 40 cycles. $(20 \mu \mathrm{L})$ consisted of $6.4 \mu \mathrm{L}$ of $\mathrm{dH} 2 \mathrm{O}, 1.6 \mu \mathrm{L}$ of gene-specific primer $(10 \mu \mathrm{M})$, $2 \mu \mathrm{L}$ of synthesized cDNA, and $10 \mu \mathrm{L}$ of SYBR Premix Ex $\mathrm{Taq}^{\mathrm{TM}} \mathrm{II}$ in the PCR reaction mixture. $2^{-\Delta \Delta \mathrm{Ct}}$ method was set to compute comparative declaration. \& the Ct value of U6 was set as the inner reference.

\section{Determining plasma levels cTnI and CK-MB}

Plasma CK-MB \& cTnI levels were determined using their respective ELISA kits. 
Table 1 Clinicopathological characteristics of patients

\begin{tabular}{lccc}
\hline Parameter & Control $(\mathrm{n}=40)$ & AMI $(\mathrm{n}=80)$ & $\mathrm{P}$ \\
\hline Male & $12(30 \%)$ & $20(25 \%)$ & 0.6621 \\
Age (years) & $65.40 \pm 5.80$ & $66.90 \pm 7.80$ & 0.2842 \\
Smoking & $11(27.5 \%)$ & $15(18.7 \%)$ & 0.3475 \\
Hypertension & $10(12.5 \%)$ & $28(35 \%)$ & 0.3037 \\
TC (mmol/L) & $3.79 \pm 1.08$ & $4.15 \pm 1.15$ & 0.1018 \\
TG (mmol/L) & $1.45 \pm 1.11$ & $1.79 \pm 0.97$ & 0.0873 \\
HDL-C (mmol/L) & $1.21 \pm 0.51$ & $1.15 \pm 0.87$ & 0.6881 \\
LDL-C (mmol/L) & $2.31 \pm 1.03$ & $2.74 \pm 1.23$ & 0.0597 \\
\hline
\end{tabular}

LDL-C, low-density lipoprotein cholesterol; HDL-C, high-density lipoprotein cholesterol; TG, triacylglycerol; TC, total cholesterol; $\mathrm{AMI}$, acute myocardial infraction.

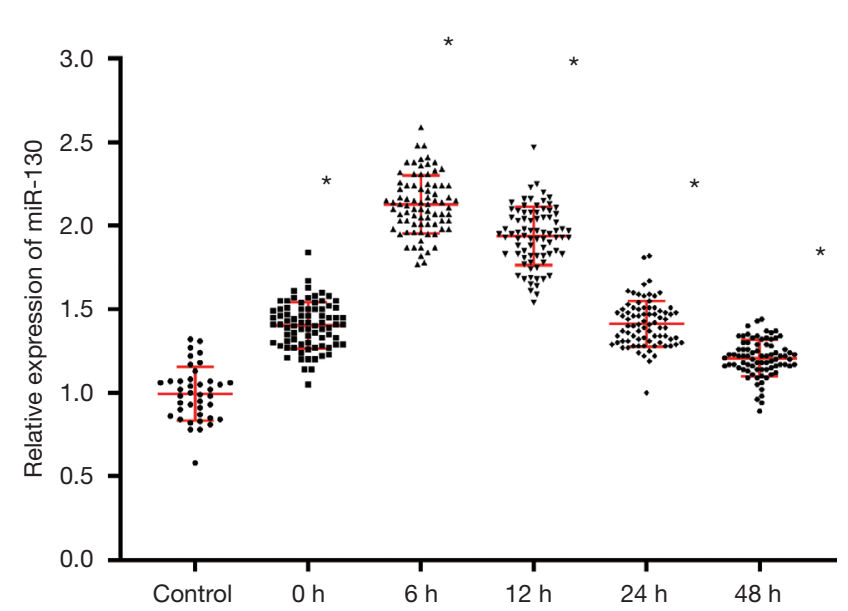

Figure 1 Level of expression of miR-130 in patient plasma. ${ }^{*}, \mathrm{P}<0.05$, in contrast with power grouping.

\section{Determination of diagnostic value of miR-130}

Diagnostic performance of miR-130 was measured using a receiver operating characteristic (ROC) curve.

\section{Arithmetical examination}

Statistical analysis performed using SPSS (19.0). Data expressed as mean \pm SD. Classifications differentiated using a Chi-squared trial. Correlation analysis carried out by using Spearman's rank correlation coefficient. Arithmetical importance was presume at $\mathrm{P}<0.05$.

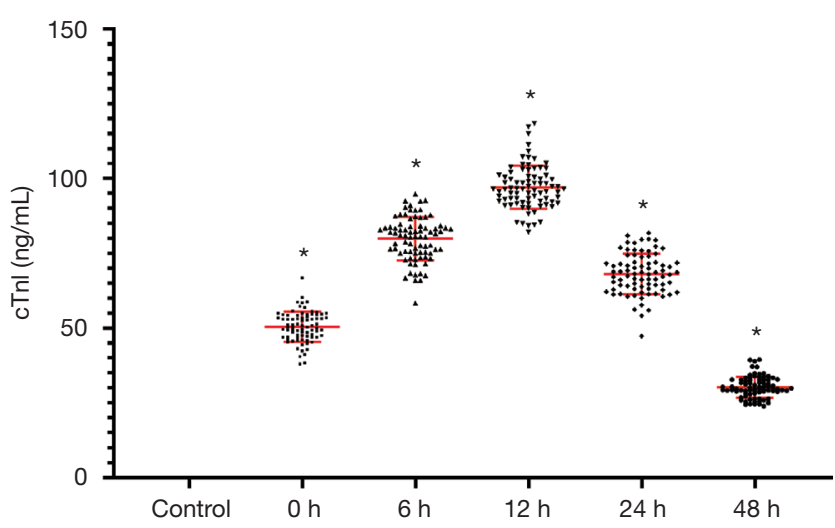

Figure 2 Plasma cardiac troponin I (cTnI) levels in acute myocardial infarction (AMI) and control patients. *, $\mathrm{P}<0.05$, in contrast with power grouping.

\section{Results \\ Clinicopathological characteristics of patients}

No significant distinction in patients' clinic pathological attribute between the two categories $(\mathrm{P}<0.05$; Table 1).

\section{Level of expression of miR-130 in plasma}

As we can see in Figure 1, the expression extent of miR130 is seriously elevated in the AMI classification of patients compared to the control group $(\mathrm{P}<0.05)$. MiR-130 expression peaked 6 hours after disease onset.

\section{Plasma cTnI levels in AMI and control patients}

Plasma cTnI levels are noticeably uplifted in the AMI class than in the control category $(\mathrm{P}<0.05)$. The levels of $\mathrm{c} T \mathrm{Tn}$ peaked 12 hours after disease onset. These results as shown in Figure 2.

\section{Plasma CK-MB levels in AMI and control patients}

Plasma CK-MB levels are remarkably going higher in the AMI group in comparison with the control grouping $(\mathrm{P}<0.05)$, and levels peaked 12 hours after disease onset (Figure 3).

\section{Correlation between miR-130, cTnI, and CK-MB levels}

As we can find in Figure 4. The assertion amount of miR-130 


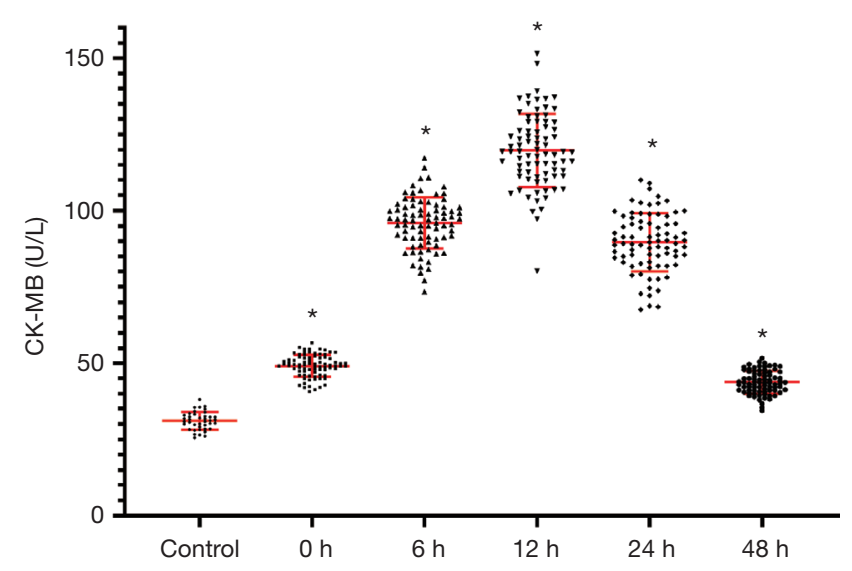

Figure 3 Plasma creatine phosphokinase (CPK)-MB amount in cardiac infarction \& control cases. *, $\mathrm{P}<0.05$, in contrast with control grouping.

6 hours after disease onset was positively correlate with cTnI and CK-MB levels 12 hours after onset.

\section{Diagnostic value of miR-130 in AMI patients}

The optimal cut-off point for miR-130 in peripheral blood, sensitivity, and specificity were $1.58 \mathrm{ng} / \mathrm{mL}, 82.5 \%$ and $77.5 \%$, respectively. The area under the curve (AUC) was 0.922 (Figure 5).

\section{Discussion}

When arteries to the heart muscle interrupted, acute myocardial infarction becomes a hazardous situation, originate harm to the tissues. Typically blockage in one or more of the coronary vessels. Indications include fatigue, lightheadedness, abnormal heartbeat, anxiety, as well as chest cramp or heartburn \& agony in back, arms or neck. Males has fewer chances than female for unusual prodrome.

The regimen covers medication \& lifestyle changes. Cardiac rehabilitation, bypass surgery \& stents. AMI is predominantly caused by CAD. The probability elements incorporate soaring arterial pressure, Diabetic ketoacidosis, smoking, sedentary lifestyle, heaviness, soaring arterial dietary fat, immoderate liquor depletion \& imperfect edibles. Absolute obstruction of a cardiac vessel due to severance in atherosclerotic plaque is normally the fundamental appliance in AMI.

MiRNAs are complicated in carrying over synchronization of mRNAs. MiRNA gene are demonstrated as centrally reproduction (pri-miRNA) that is eventually prepared to grownup miRNAs through forerunner miRNAs (premiRNA). The adult miRNAs form incomplete nucleotide named bud go with selected mRNAs at 3'UTR of selected gene (17). MiRNAs have been identified as attractive targets for therapeutic intervention in cardiac pathologies, but the underlying mechanism of miRNAs in AMI remains largely unknown. The miRNA-130 gene is located on chromosome $11 \mathrm{q} 12.1$. Its action is mediated by bone morphogenetic protein 2 (BMP-2), a component of the FGF8 signaling pathway (17). The expression of miR-130 is downregulated in obese hearts, suggestive of a role in ischemia-reperfusion injury or AMI (18). The dysregulation of miR-130 in macrovesicles is associated with cardiovascular events in patients with CAD (19). MiRNA-130 has also been shown to promote hypoxia-induced smooth muscle cell proliferation, thereby contributing to vascular remodeling in pulmonary hypertension and right ventricular hypertrophy (20). Recent studies have shown that miR-130 expression is significantly upregulated thereafter-remaining preceding dropping coronary tubal sterilization. In H9c2 cells, miR-130 aggravates AMI-induced injury by targeting PPAR- $\gamma$ (15). This study investigated the diagnostic value of miR-130 in patients with AMI. The results showed that miR-130 was significantly upregulated in AMI, indicating potential as a biomarker for the disease.

The usual biochemical markers for AMI are cTnI, cTnT, CK-MB, and myoglobin (21). However, their clinical applications for the early diagnosis of AMI have produced unsatisfactory results. Both $\mathrm{c} T n \mathrm{~T}$ and $c \operatorname{Tn} I$ are heart legislative oligopeptides, which administer chemicalcompound arbitrate. Relation allying myosin \& actin. Particular genes code the coronary shape of these legislative peptides. That has the latent of being distinctive to the cardiac muscle. In particular, $c \operatorname{TnI}$ is extensively used in diagnosis of AMI (7). The enzyme CK-MB is one of the isoforms of CK. It is derived from the myocardium, and serves as a key diagnostic index in patients with $\operatorname{AMI}(22,23)$. The circulating levels of cTnI and CK-MB are elevated 3 to 6 hours after AMI onset, and return to normal after 3 to 4 days, and 11 to 14 days after onset, respectively (24). In this study, the levels of cTnI \& CK-MB betide markedly elevated the (acute myocardial infarction) group, less than the control group, and peaked 12 hours after disease onset. The expression level in miR-130 has also seriously upregulated in AMI class, relative to administer classification \& the levels peaked 6 hours after AMI onset. The circulating levels of miR-130 peaked earlier relatively cTnI and CK-MB, an indication that miR-130 
A

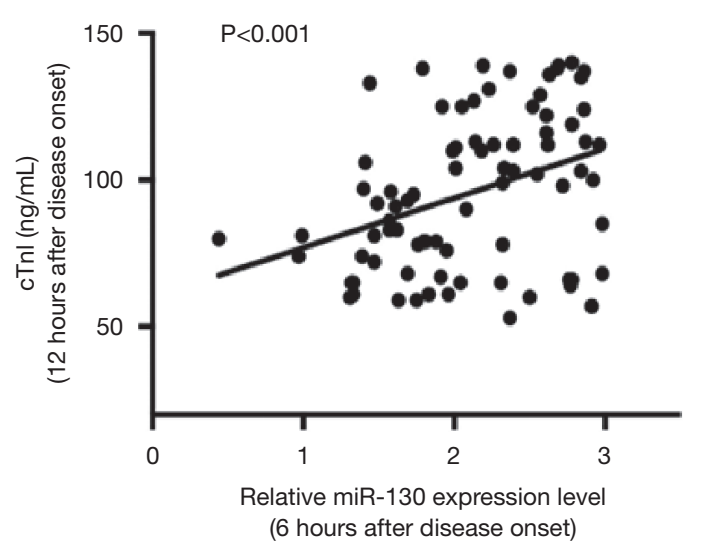

B

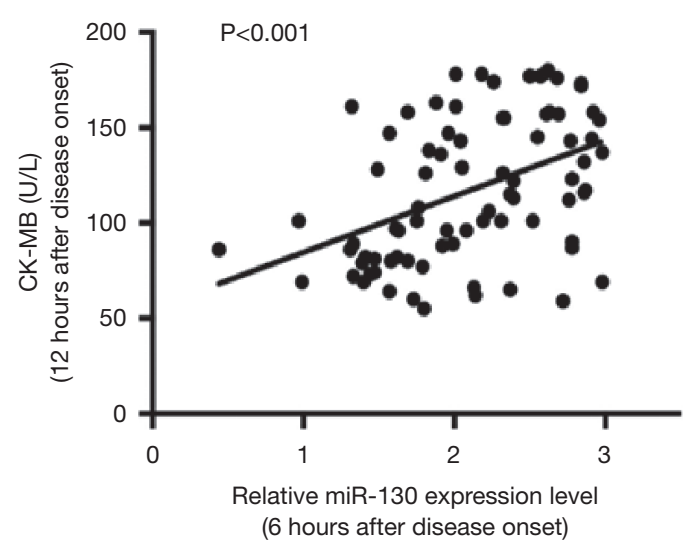

Figure 4 Correlation between miR-130 (6 hours after disease onset) and cardiac troponin I (cTnI) (12 hours after onset), creatine kinase (CK)-MB (12 hours after onset) levels in acute myocardial infarction (AMI) patients.

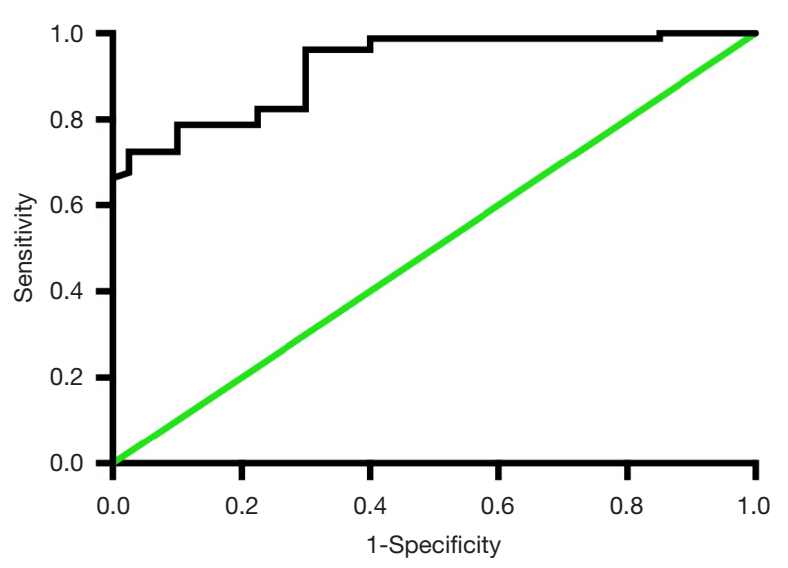

Figure 5 The diagnostic value of miR-130 in acute myocardial infarction (AMI) patients.

could be useful for earlier detection in AMI. Therefore, consequences of this investigation suggest that circulating miR-130 levels may increase the diagnostic accuracy of cTnI and CK-MB, \& may set out a useful guide towards earlier detection in AMI.

\section{Conclusions}

Circulating miR-130 holds great promise as an effective biomarker for the early diagnosis of AMI.

\section{Acknowledgments}

Funding: This work was supported by a grant from the
National Natural Science Foundation of China (No. 81500204).

\section{Footnote}

Reporting Checklist: The authors have completed the STARD reporting checklist. Available at http://dx.doi.org/10.21037/ jtd-20-3207

Data Sharing Statement: Available at http://dx.doi. org/10.21037/jtd-20-3207

Conflicts of Interest: All authors have completed the ICMJE uniform disclosure form (available at http://dx.doi. org/10.21037/jtd-20-3207). The authors have no conflicts of interest to declare.

Ethical Statement: The authors are accountable for all aspects of the work in ensuring that questions related to the accuracy or integrity of any part of the work are appropriately investigated and resolved. All procedures performed in this study involving human participants were in accordance with the Declaration of Helsinki (as revised in 2013). The study protocol was approved by the Clinical Research Ethics Committee of Zhongda Hospital Affiliated to Southeast University, China. The patients signed written informed consent with their family members.

Open Access Statement: This is an Open Access article distributed in accordance with the Creative Commons 
Attribution-NonCommercial-NoDerivs 4.0 International License (CC BY-NC-ND 4.0), which permits the noncommercial replication and distribution of the article with the strict proviso that no changes or edits are made and the original work is properly cited (including links to both the formal publication through the relevant DOI and the license). See: https://creativecommons.org/licenses/by-nc-nd/4.0/.

\section{References}

1. Gabriel-Costa D. The pathophysiology of myocardial infarction-induced heart failure. Pathophysiology 2018;25:277-84.

2. Wang C, Jing Q. Non-coding RNAs as biomarkers for acute myocardial infarction. Acta Pharmacol Sin 2018;39:1110-9.

3. Xu S, Jiang J, Zhang Y, et al. Discovery of potential plasma protein biomarkers for acute myocardial infarction via proteomics. J Thorac Dis 2019;11:3962-72.

4. Prastaro M, Pirozzi E, Gaibazzi N, et al. Expert Review on the Prognostic Role of Echocardiography after Acute Myocardial Infarction. J Am Soc Echocardiogr 2017;30:431-43.e2.

5. Vafaie M. State-of-the-art diagnosis of myocardial infarction. Diagnosis 2016;3:137-42.

6. Apple FS, Sandoval Y, Jaffe AS, et al. Cardiac Troponin Assays: Guide to Understanding Analytical Characteristics and Their Impact on Clinical Care. Clin Chem 2017;63:73-81.

7. Westermann D, Neumann JT, Sorensen NA, et al. Highsensitivity assays for troponin in patients with cardiac disease. Nat Rev Cardiol 2017;14:472-83.

8. Yang $X, S u W$, Chen $X$, et al. Validation of a serum 4-microRNA signature for the detection of lung cancer. Transl Lung Cancer Res 2019;8:636-48.

9. Hinkel R, Ng JK, Kupatt C. Targeting microRNAs for cardiovascular therapeutics in coronary artery disease. Curr Opin Cardiol 2014;29:586-94.

10. Paiva S, Agbulut O. MiRroring the Multiple Potentials of MicroRNAs in Acute Myocardial Infarction. Front Cardiovasc Med 2017;4:73.

11. Cheng Y, Tan N, Yang J, et al. A translational study of circulating cell-free microRNA-1 in acute myocardial infarction. Clin Sci (Lond) 2010;119:87-95.

12. Zhong J, He Y, Chen W, et al. Circulating microRNA$19 \mathrm{a}$ as a potential novel biomarker for diagnosis of acute myocardial infarction. Int J Mol Sci 2014;15:20355-64.

13. Zhang L, Chen X, Su T, et al. Circulating miR-499 are novel and sensitive biomarker of acute myocardial infarction. J Thorac Dis 2015;7:303-8.

14. Liu X, Yuan L, Chen F, et al. Circulating miR-208b: A Potentially Sensitive and Reliable Biomarker for the Diagnosis and Prognosis of Acute Myocardial Infarction. Clin Lab 2017;63:101-9.

15. Chu X, Wang Y, Pang L, et al. miR-130 aggravates acute myocardial infarction-induced myocardial injury by targeting PPAR-gamma. J Cell Biochem 2018;119:7235-44.

16. Thygesen K, Alpert JS, White HD, et al. Universal definition of myocardial infarction. Circulation 2007;116:2634-53.

17. Lopez-Sanchez C, Franco D, Bonet F, et al. Negative Fgf8-Bmp2 feed-back is regulated by miR-130 during early cardiac specification. Dev Biol 2015;406:63-73.

18. Sassoon DJ, Goodwill AG, Noblet JN, et al. Obesity alters molecular and functional cardiac responses to ischemia/ reperfusion and glucagon-like peptide-1 receptor agonism. Basic Res Cardiol 2016;111:43.

19. Jansen F, Yang X, Proebsting S, et al. MicroRNA expression in circulating microvesicles predicts cardiovascular events in patients with coronary artery disease. J Am Heart Assoc 2014;3:e001249.

20. Brock M, Haider TJ, Vogel J, et al. The hypoxia-induced microRNA-130a controls pulmonary smooth muscle cell proliferation by directly targeting CDKN1A. Int J Biochem Cell Biol 2015;61:129-37.

21. Danese E, Montagnana M. An historical approach to the diagnostic biomarkers of acute coronary syndrome. Ann Transl Med 2016;4:194.

22. Jang JS, Jin HY, Seo JS, et al. Prognostic value of creatine kinase-myocardial band isoenzyme elevation following percutaneous coronary intervention: a meta-analysis. Catheter Cardiovasc Interv 2013;81:959-67.

23. Kehl DW, Iqbal N, Fard A, et al. Biomarkers in acute myocardial injury. Transl Res 2012;159:252-64.

24. Guo ML, Guo LL, Weng YQ. Implication of peripheral blood miRNA-124 in predicting acute myocardial infarction. Eur Rev Med Pharmacol Sci 2017;21:1054-9.

(English Language Editor: C. Betlazar-Maseh)

Cite this article as: Pan X, He Y, Chen Z, Yan G, Ma G. Circulating miR-130 is a potential bio signature for early prognosis of acute myocardial infarction. J Thorac Dis 2020;12(12):7320-7325. doi: 10.21037/jtd-20-3207 